\title{
THE EMERGENCE OF A BLACK HOLE IN SUPERNOVA EXPLOSIONS
}

\author{
L. Zampieri*, M. Colpi ${ }^{\dagger}$, S. Shapiro*,††, and I. Wasserman ${ }^{\dagger \dagger}$ \\ * Department of Physics, University of Illinois at Urbana-Champaign, Urbana, IL 61801 \\ ${ }^{\dagger}$ Dipartimento di Fisica, Università degli Studi di Milano, I-20133 Milano, Italy \\ ${ }^{\dagger \dagger}$ Department of Astronomy and National Center for Supercomputing Applications, University of \\ Illinois at Urbana-Champaign, Urbana, IL 61801 \\ ${ }^{\dagger \dagger}$ Center for Radiophysics and Space Research, Cornell University, Ithaca, NY 14853
}

\begin{abstract}
.
We present results from a fully relativistic investigation of matter fallback in a supernova, assuming that the central compact star is a black hole (BH). Our goal is to answer the following questions: can we infer from the light curve whether a stellar black hole (BH) has formed in the aftermath of a supernova explosion ? Did SN1897A produce a $\mathrm{BH}$ and, if so, when will the hole become detectable ? We show that it should not be possible to infer the presence of a $\mathrm{BH}$ in the remnant for another several hundred to a thousand years. In the case of SN1987A, we estimate the BH luminosity to be $L \simeq 5 \times 10^{34} \mathrm{erg} \mathrm{s}^{-1}$, which is well below the present day bolometric luminosity of the remnant $\left(\sim 10^{36} \mathrm{erg} \mathrm{s}^{-1}\right.$; Suntzeff $\left.1997[1]\right)$.
\end{abstract}

\section{INTRODUCTION}

Stellar evolution calculations show that stars with a main sequence mass in the range 8-19 $M_{\odot}$ finish their lives with a compact core of $\sim 1.4 M_{\odot}$ (Woosley \& Weaver 1995 [2]). For these stars the amount of material that falls back toward the core in the aftermath of its collapse is negligible, so it is likely that they give birth to neutron stars. The fate of stars with main sequence mass in the range 19-25 $M_{\odot}$ is far less obvious. At the end of their evolution, they have core masses around 1.6-1.8 $M_{\odot}$ and a variable amount of matter, 0.1-0.3 $M_{\odot}$ may fall back. Typically, these explosions leave a central compact remnant of 1.7-2.1 $M_{\odot}$ whose nature depends critically on the maximum (gravitational) mass of a neutron star, $M_{c r i t}$, and hence on the equation of state of dense nuclear matter. To date, the value of $M_{\text {crit }}$ is quite uncertain: $1.5 M_{\odot}<M_{\text {crit }}<2.5 M_{\odot}$ for a nonrotating star.

The mass of the progenitor of SN1987A $\left(M=18-21 M_{\odot}\right)$ falls in the range of masses where the outcome of core collapse is uncertain; from a theoretical point of view, we do not know if a neutron star or a black hole formed during the explosion. However, if a compact object of any kind is present, it is probably accreting from 
the progenitor stellar material (Chevalier 1989 [3]). The bolometric light curve observed to date can be explained by the "standard" theory of Type II supernovae. It is therefore important to determine what extra luminosity an accreting central component would produce and when its presence might be discernible. In this paper we present results from the first fully relativistic investigation of supernova fallback in presence of a $\mathrm{BH}$ including the effects of hydrodynamics and radiation transport.

\section{METHOD AND NUMERICAL RESULTS}

We constructed a spherically symmetric, general-relativistic radiation hydrodynamic Lagrangian code capable of handling the transfer of radiation from the early phase when photons diffuse through a high temperature, expanding cloud, to the late stage when the hydrogen envelope has recombined and most of the ejecta are transparent (Zampieri et al. (1997) [4]). We consider fallback from a hydrogen "cloud"; all of the emitted energy comes from the release of heat residing in the gas originally, or is generated by compressional heating in the course of accretion onto the BH. Radioactive energy sources have not been included in our calculations. At the onset of evolution the cloud has homogenous density $\rho_{0}$ and a velocity profile which scales linearly with radius $r$. The temperature profile is taken to be equal to the "radiative zero solution" given by Arnett (1980) [5] and the degree of ionization is computed throughout evolution according to the Saha equation. Four parameters uniquely specify the state of the "cloud": the total mass $M_{\text {cloud }}$, the radius $r_{\text {out }}$, the sound speed at the inner boundary $c_{s, 0}$, and the ratio $\tilde{k}$ of the accretion timescale $t_{a, 0}=G M_{b h} / c_{a, 0}^{3}\left(M_{b h}\right.$ mass of the $\left.\mathrm{BH}\right)$ to the expansion time $t_{0}=r_{\text {out }} / V_{0}\left(V_{0}\right.$ maximum expansion velocity of the ejecta).

We find two phases of evolution. In the first stage, gas expands adiabatically in the outer region and accretes close to the central hole. During this phase, the cloud is radiation dominated. A second phase follows, during which hydrogen recombines and the "cloud" becomes optically thin. Fig. 1 shows the temperature profile of model A characterized by $M_{\text {cloud }}=1 M_{\odot}\left(\rho_{0}=2 \times 10^{-5} \mathrm{~g} \mathrm{~cm}^{-3}\right), r_{\text {out }}=$ $3 \times 10^{12} \mathrm{~cm}, \tilde{k}=0.1$ and $c_{s, 0}=10^{8} \mathrm{~cm} \mathrm{~s}^{-1}$. A recombination wave develops that propagates rapidly through the envelope. The recombination front stalls as soon as it approaches the innermost region where compressional heating due to accretion overcomes radiative cooling. Outside the recombination front the flow is transparent but it remains optically thick inside for a long time. The density and velocity profiles display a self-similar character and are unaffected by the propagation of the recombination front.

As recombination begins, the huge amount of internal energy released gives rise to a big bump in the light curve at $t \sim 10$ days, as illustrated in Fig. 2. After the emission maximum, the luminosity falls off abruptly. During the late evolution, the light curve is entirely powered by accretion onto the central $\mathrm{BH}$ and the luminosity

decreases as a power law with time. At late times, the evolution proceeds through 


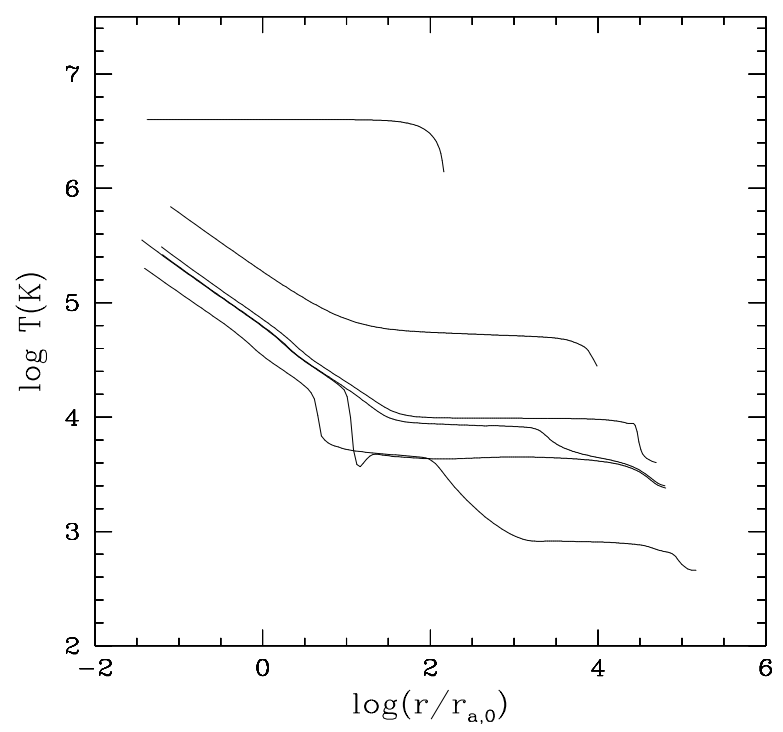

FIGURE 1. Gas temperature $T$ versus radius $r$ (in units of the initial accretion radius $\left.r_{a, 0}=G M_{b h} / c_{s, 0}^{2}\right)$ at selected times for model A. From the top to the bottom: $t / t_{a, 0}=0$, $729,3757,4755,4870,11175$.

a sequence of quasistationary states, and $\dot{m}$ (in Eddington units) can be estimated using the scaling relation for a pressureless fluid (Colpi et al. 1996 [6]; equation [29])

$$
\dot{m}(t) \simeq\left[4 \pi^{2 / 3} / 9\right] \rho_{0} t_{0} k_{e s} c\left(t / t_{0}\right)^{-5 / 3},
$$

where $k_{e s}$ is the electron scattering opacity. The luminosity $l$ (in Eddington units) is close to that derived by Blondin (1986) [7] for stationary accretion

$$
l \simeq 3 \times 10^{-7}\left(M_{b h} / M_{\odot}\right)^{-1 / 3} \dot{m}^{5 / 6} .
$$

In Fig. 2, the dotted line at the bottom denotes the late-time accretion luminosity estimated using equations (1) and (2): the analytic extrapolation is in good agreement with the computed light curve.

\section{THE EMERGENCE OF A BH IN SN1987A}

Slightly after maximum, SN1987A was powered by the radioactive decay of ${ }^{56} \mathrm{Co}$ and ${ }^{57} \mathrm{Co}$, and at present its light curve is consistent with emission from the decay of ${ }^{44} \mathrm{Ti}$ (Suntzeff 1997 [1]; Fig. 2). Can we discern the luminosity emitted by the accreting $\mathrm{BH}$ above the contribution resulting from ${ }^{44} \mathrm{Ti}$ ? An upper limit to the accretion luminosity of SN1987A can be inferred from equations (1) and (2) (for details see Zampieri et al. (1997) [4]). Following Chevalier (1989) [3], we adopt $\rho_{0} t_{0}^{3} \approx 10^{9} \operatorname{cgs}$ and $t_{0} \approx 7000$ seconds, yielding a luminosity 


$$
l \simeq \frac{8 \times 10^{-3}}{\left(M_{b h} / M_{\odot}\right)^{1 / 3}[t(\text { years })]^{25 / 18}} .
$$

Equation (3) implies $L \simeq 5 \times 10^{34} \mathrm{erg} \mathrm{s}^{-1}$ after $t \approx 10$ years, which is well below the present day bolometric luminosity of the remnant $\left(\sim 10^{36} \mathrm{erg} \mathrm{s}^{-1}\right.$; Suntzeff $\left.1997[1]\right)$ and also smaller than the luminosity estimated to result from radioactive decay. Thus, there is no observation that rules out the possibility that a $\mathrm{BH}$ resides inside the SN1987A remnant. In Fig. 2 we plot the late-time light curve of SN1987A calculated using (3). As radioactive decay plummets at around 270-2700 years, it is clear that the $\mathrm{BH}$ would only appear after about 900 years irrespective of the detailed numbers. After this time has elapsed, the luminosity of the remnant would be $\sim 10^{32} \mathrm{erg} \mathrm{s}^{-1}$, too dim for detection with present technology. The inner part of the accretion flow will be still optically thick and will be emitting roughly a black body spectrum peaked in the visible band.

\section{REFERENCES}

1. Suntzeff, N.B. 1997, in SN1987A: Ten Years After, ed. M.M. Phillips and N.B. Suntzeff (ASP Conference Series)

2. Woosley, S.E., \& Weaver T.A. 1995, ApJS, 101, 181

3. Chevalier, R.A. 1989, ApJ, 346, 847

4. Zampieri, L., Colpi, M., Shapiro, S.L., Wasserman, I. 1997, submitted to ApJ

5. Arnett, D. 1980, ApJ, 237, 541

6. Colpi, M., Shapiro, S.L., \& Wasserman, I. 1996, ApJ, 470, 1075

7. Blondin, J.M. 1986, ApJ, 308, 755 


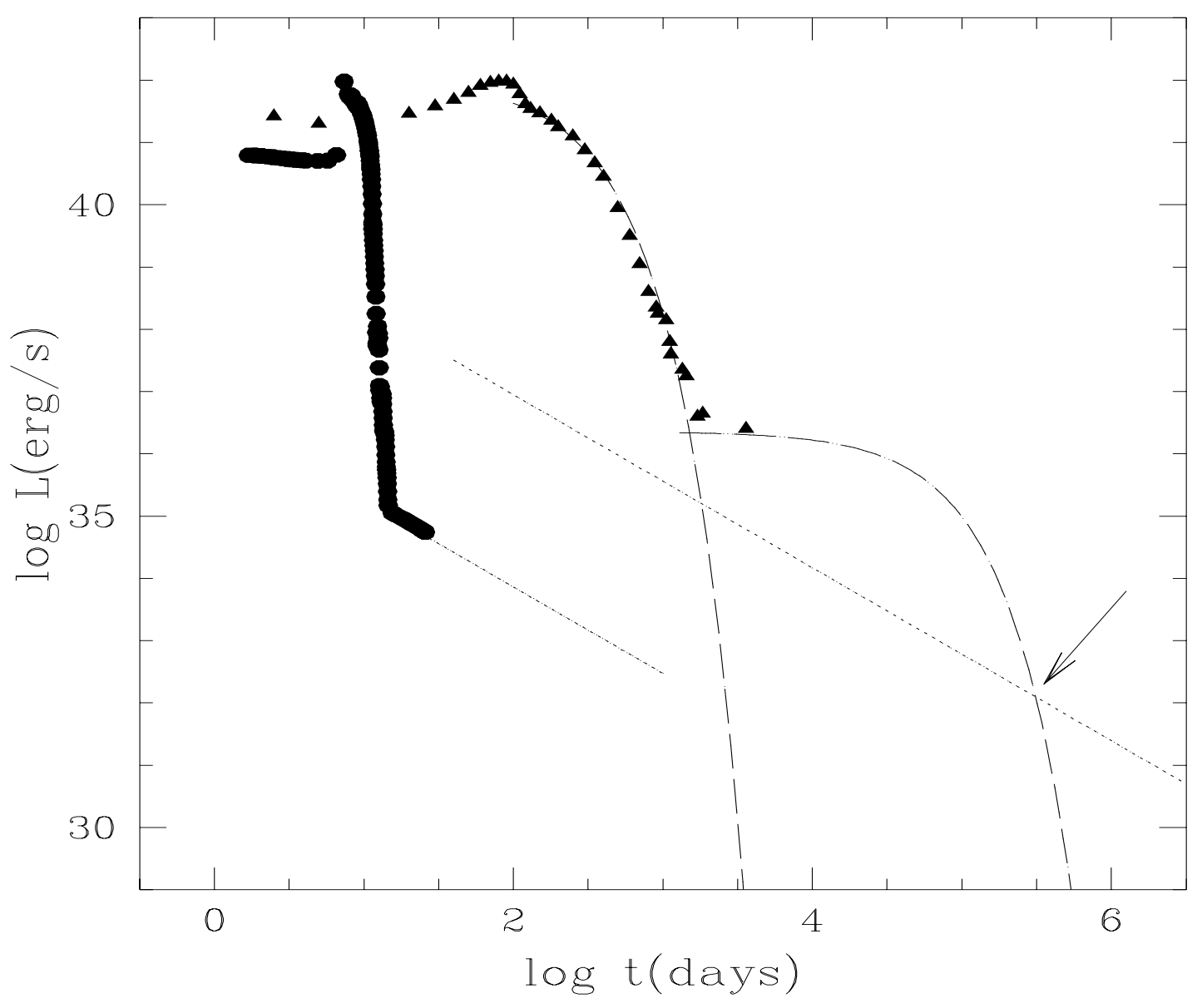

FIGURE 2. The Circles give the solution for model A. The Dotted line is the extrapolation of the late-time evolution. The Triangles are the bolometric luminosity of SN1987A. The Dashed lines represent the contribution from the decay of radioactive elements $\left(0.07 M_{\odot}\right.$ of ${ }^{56} \mathrm{Co}$ and $\sim 5 \times 10^{-5} M_{\odot}$ of $\left.{ }^{44} \mathrm{Ti}\right)$. Finally, the upper dotted line denotes the expected bolometric luminosity emitted by a putative BH in SN1987A (equation [3]). The arrow marks the time ( $t \simeq 900$ years) of the $\mathrm{BH}$ emergence. 